\title{
Computed tomography angiography-based analysis of high-risk intracerebral haemorrhage patients by employing a mathematical model
}

\author{
Le Zhang ${ }^{1,2,3^{*}}$, Jin $\mathrm{Li}^{1,4}$, Kaikai Yin ${ }^{1}$, Zhouyang Jiang ${ }^{5}$, Tingting $\mathrm{Li}^{6}$, Rong $\mathrm{Hu}^{5}$, Zheng $\mathrm{Yu}^{7}$, Hua Feng ${ }^{5}$ and
} Yujie Chen ${ }^{5^{*}}$

From The 12th International Conference on Computational Systems Biology (ISB 2018)

Guiyang, China. 18-21 August 2018

\begin{abstract}
Background: Haemorrhagic stroke accounts for approximately $31.52 \%$ of all stroke cases, and the most common origin is hypertension. However, little is known about the method to identify high-risk populations of hypertensive intracerebral haemorrhage.

Results: The results showed that the angle between the middle cerebral artery and the internal carotid artery (AMIC), the distance between the beginning of the median artery and superior trunk (DMS), and the density (CT value) of the lenticulostriate artery $(\mathrm{CTL})$ were statistically significant enough to cause intracerebral haemorrhage. In addition, we chose these three potential features for the ensemble learning classification model. Our developed ensemble-learning method outperforms not only previous work but also three other classic classification methods based on accuracy measurements.

Conclusions: The developed mathematical model in the present study is efficient in predicting the probability of intracerebral haemorrhage.
\end{abstract}

Keywords: Intracerebral haemorrhage, Computed tomography angiography, Ensemble learning, Lenticulostriate arterial, data mining

\section{Background}

Haemorrhagic stroke accounts for approximately $31.52 \%$ of all strokes, and the most common origin is hypertension [1]. The most frequent location of hypertensive intracerebral haemorrhage is around the basal ganglia and thalamus, which could easily lead to death or disability [2]. The most prevalent risk factors among stroke survivors are hypertension (88\%), smoking (48\%), and alcohol use (44\%) [3]. However, we know little about the method

\footnotetext{
* Correspondence: zhangle06@scu.edu.cn; yujiechen6886@foxmail.com ${ }^{1}$ College of Computer and Information Science, Southwest University, Chongqing 400715, People's Republic of China

${ }^{5}$ Department of Neurosurgery, Southwest Hospital, Third Military Medical University, Chongqing 400038, People's Republic of China

Full list of author information is available at the end of the article
}

to identify high-risk populations of hypertensive intracerebral haemorrhage. Since the pre-diagnosis of intracerebral haemorrhage can effectively reduce the incidence rate of intracerebral haemorrhage [4-8], this research develops a mathematical model for the prediction of the probability of intracerebral haemorrhage.

Recently, computational biologists employed information technologies to predict haematoma expansion after intracerebral haemorrhage and the prognosis of intracerebral haemorrhage [9-11], but only a few studies consider the haemorrhage risk for the non-intracerebral haemorrhage patients. Previous studies [12, 13] already used external factors (i.e., temperature and weather $[12,13]$ ) as the features to build up the predictive models for

(c) The Author(s). 2019 Open Access This article is distributed under the terms of the Creative Commons Attribution 4.0 International License (http://creativecommons.org/licenses/by/4.0/), which permits unrestricted use, distribution, and 
intracerebral haemorrhage. However, these models can only predict population occurrence probability for the intracerebral haemorrhage, and it is difficult for us to predict the probability of intracerebral haemorrhage and obtain high predictive accuracy for each patient.

Usually, we employ computed tomography (CT) images to predict the haematoma enlargement after intracerebral haemorrhage, or the historical characteristics and biomarkers for the neurological outcome prediction of intracerebral haemorrhage patients. Recently, computational biologists started using density, area and other factors of the abnormal area of haematoma in CT images to develop a predictive model for haematoma enlargement after intracerebral haemorrhage $[14,15]$. However, since recent studies could not collect adequate training and testing data, they only predicted the degree of deterioration of intracerebral haemorrhage but could not effectively estimate the probability of intracerebral haemorrhage for these patients.

To overcome the shortcomings for previous predictive models $[5-8,10,11]$, this study proposes a mathematical framework for the prediction of intracerebral haemorrhage with the following three innovations. First, statistical methods are employed to compute the size of the samples and demonstrate which candidate biomarkers are statistically significant enough to be the features of the model. Second, the predictive model is built with these features. Finally, a novel ensemble-learning method [16] is developed by integrating the support vector machine (SVM) [17], decision tree [18] and K-Nearest Neighbor(KNN) [19] algorithms into the model to improve the predictive accuracy.

Our research results reveal that AMIC, DMS and CTL can predict intracerebral haemorrhage. Moreover, we use them to build the ensemble-learning predictive model, which not only outperforms the classic SVM, decision tree and KNN models but also performs better than previous similar research [20-22].

\section{Methods}

\section{Experimental materials}

A total of 151 patients with unilateral hypertensive ICH admitted to the Department of Neurosurgery, Southwest Hospital, Chongqing, China and confirmed with computed tomography angiography between January 2012 and December 2016 were analysed retrospectively. The protocol was approved by the Ethics Committee of Southwest Hospital, and the committee waived the need for patient consent due to the retrospective nature of the study. The basic information of these patients is listed in Additional file 1: Tables S1 and Table S2. All patients' computed tomography angiography images were analysed from the haematoma side and non-haematoma side as a self-controlled study of the high-risk features of intracerebral haemorrhage.

Since haemodynamics around the middle cerebral artery and lenticulostriate artery are tightly associated with the blood vessel rupture for intracerebral haemorrhagic patients [23-25], we selected ten potential features (Fig. 1) related to the artery angle, artery distance, and artery density as the candidate features for model development in this area (Additional file 1: Table S3).

This study recruited $151 \mathrm{CT}$ angiography imaging series, and we used RadidaDicomViewer software [26] to extract the digital values of the potential features (Additional file 1: Table S4) from the samples.

\section{Model development}

Figure 2 shows the workflow of the mathematical model. The first step of the study was using the experimental design to determine the size of the sample and employing statistical tests to choose the candidate features for the model from the potential features. Second, we developed a novel ensemble-learning method by integrating SVM, the decision tree and $\mathrm{KNN}$ algorithm into our predictive model based on the selected features for intracerebral haemorrhage prediction. Finally, we trained and tested the model by optimizing the key parameters and validating the model predictive capacity, respectively.

\section{Sample size estimation}

Limited by resources and ethical reasons, it is impossible to obtain an infinite sample size for the optimization of the key parameters of the model, so Eq. 1 computes the optimum sample size (n) to meet statistical significance [27].

$$
\begin{aligned}
& n=\left[\frac{2\left(u_{\alpha}+u_{\beta}\right) \sigma}{\delta}\right]^{2} \\
& d=\frac{\mu_{1}-\mu_{2}}{\sigma}=\frac{\delta}{\sigma} \\
& n=\left[\frac{2\left(u_{\alpha}+u_{\beta}\right)}{d}\right]^{2}
\end{aligned}
$$

Eq. 1.1 denotes $\sigma$ as the standard deviation; $u_{\alpha}$ and $u_{\beta}$ as the critical values of the u-test at the first type of error rate and the second type of error rate, respectively.

Eq. 1.2 defines effect size d [26] as $\delta / \sigma ; \mu_{1}$ and $\mu_{2}$ as the mean values of the disease and the control data, respectively. The first and the second type of error $\alpha$ and $\beta$ were set to 0.05 and 0.1 , respectively. Indicated by Kabacoff et al. [26], $\delta$ represents $\mu_{1}-\mu_{2}$. Then, we re-wrote Eq. 1.1 as Eq. 1.3. 

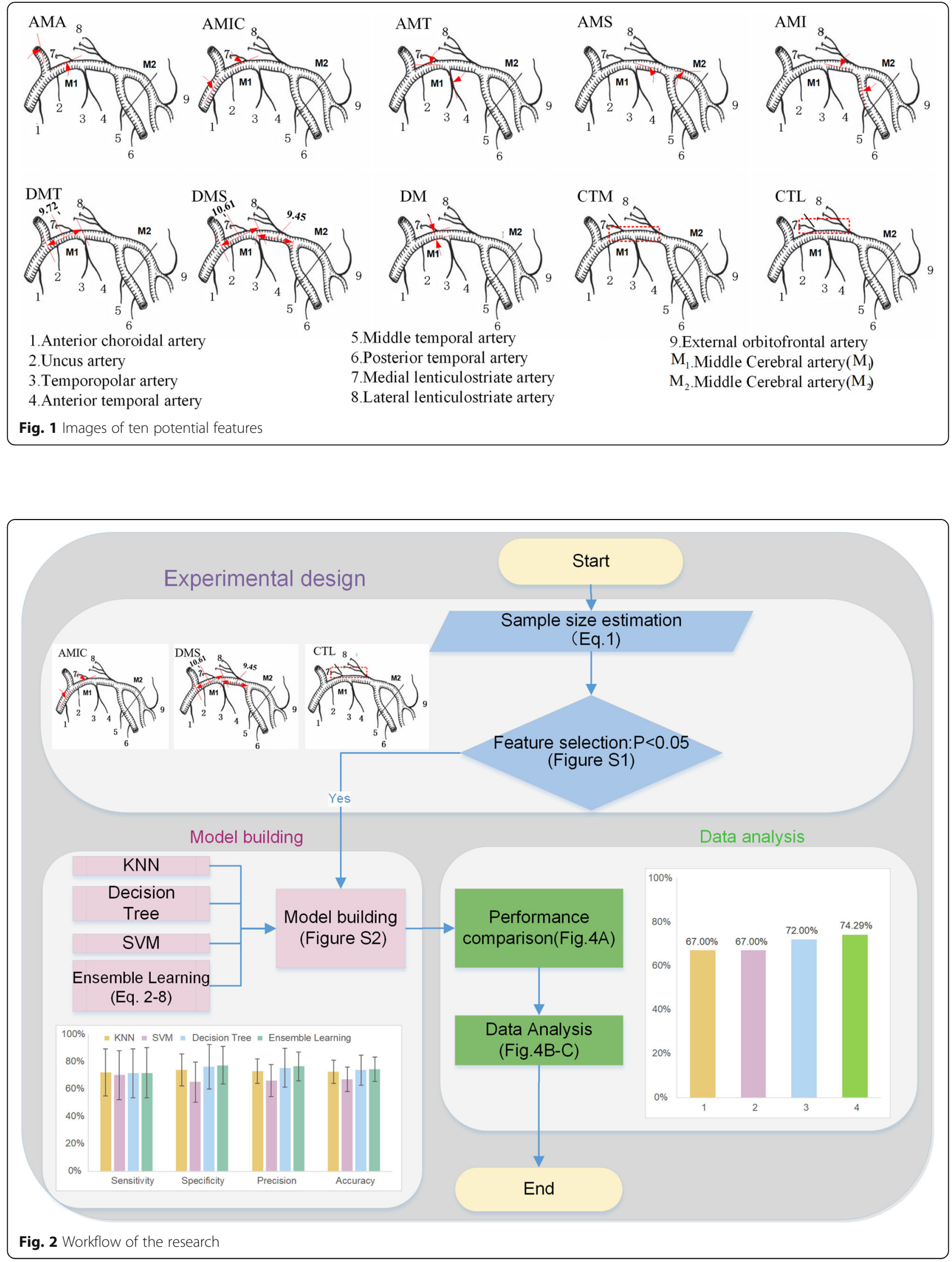


\section{Feature selection}

After locating the sample size, we employed a (Additional file 1: Figure S1) statistical significance test for these candidate features, and the digital values of this test were extracted by using RadidaDicomViewer software [26].

\section{Machine learning methods}

In the beginning, this study employed three commonly used classification algorithms [17-19] such as KNN, decision tree and SVM to develop the predictive model. Then, we integrated these three classic methods detailed in the supplemental methods section into a novel ensemble learning [16] model to improve our predictive accuracy. Here, we employed Matlab2014a [28] to implement these methods with the default parameter setup and the parameter $\mathrm{k}$ of $\mathrm{KNN}$ was set to 5 . We describe the ensemble-learning algorithm (Additional file 1: Figure S2) as follows.

Input: Sample set $S=\left\{\left(x_{1}, y_{1}\right),\left(x_{2}, y_{2}\right), \cdots,\left(x_{n}, y_{n}\right)\right\}$, where $x_{n}$ is the example and $y_{n} \in\{0,1\}$ is the label; weak classifier $\mathcal{L} \in\left\{\mathcal{L}_{1}=\mathrm{SVM}, \mathcal{L}_{2}=\right.$ Decision tree, $\mathcal{L}_{3}=\mathrm{KNN}$ \}.$T$ is the iteration number.

\section{Process:}

1) for $m=1, \ldots, \mathcal{L}$

2) Initialize the weight distribution $D_{1}(i)=1 / ;(\mathrm{n}$ is the number of examples and $i$ is the index of the example)

3) for $t=1, \ldots, T$

4) Based on the sample distribution Dt and $\mathcal{L}_{m}$, we train the weak classifier $h_{t}$

5) Compute the error $\left(\varepsilon_{t}\right)$ for $h_{t}$ $\varepsilon_{t}=\frac{\text { number of incorrectly classified examples }}{\text { total number of examples }}$

6) Compute the weight $\left(\alpha_{t}\right)$ for $h_{t}$

$\alpha_{t}=\frac{1}{2} \ln \frac{1-\varepsilon_{t}}{\varepsilon_{t}}$

7) Update the weight for each sample

$$
D_{t+1}(\mathrm{i})=\frac{D_{t}(i)}{\operatorname{sum}(D)}\left\{\begin{array}{c}
\exp \left(-\alpha_{t}\right) \text { if } h_{t}\left(x_{i}\right)=y_{i} \\
\exp \left(\alpha_{t}\right) \text { ifh }_{t}\left(x_{i}\right) \neq y_{i}
\end{array}\right.
$$

8) End

9) Obtain the ensemble learning classifier $H_{m}$ by the adaboost algorithm $[29,30]$

$$
H_{m}(x)=\operatorname{sign}(f(x))=\operatorname{sign} \sum_{t=1}^{T} \alpha_{t} h_{t}(x)
$$

10) Calculate the accuracy of $H_{m}$

$$
P_{H_{m}}=\frac{\text { number of correctly classified examples }}{\text { total number of examples }}
$$

11) End

12) Assign a weight $w_{H_{m}}$ to each $H_{m}$

$$
w_{H_{m}}=\frac{1}{2} \ln \frac{P_{H_{m}}}{1-P_{H_{m}}}
$$

Output: anomaly ensemble

$$
Y(x)=\operatorname{sign} \sum_{m=1}^{3} w_{H_{m}} H_{m}(x)
$$

\section{Results}

\section{Sample size estimation}

We used Eq. 1 to compute the sample size by setting parameter $d$ [26]. The sample size of the lower bound was 66 . Half the samples were the control and the rest were the disease.

\section{Statistical test results for the candidate features}

Since there were ten potential features closely related to the intracerebral haemorrhage $[2,4,9,12,31,32]$, this study chose them as the candidate features (Additional file 1: Table S5) to develop the predictive model. Then, we employed the statistical test workflow [33] (Additional file 1: Figure S1) to verify the statistical significance of each potential feature.

Additional file 1: Table S5 demonstrates that the angle between the middle cerebral artery and artery (AMIC), the distance between the beginning of the median cerebral artery and the superior trunk (DMS) and the CT value of the lenticulostriate artery (CTL) were statistically significant between the control and the disease data set since they have small $p$ values. To confirm these statistical results, we collected a clinical CT image to investigate these three potential features. Figure 3 shows that the angle of the AMIC was 127.7 degrees, the length of the DMS was $18.4 \mathrm{~mm}$ and the CTL was $242 \mathrm{HU}$ on the haematoma side, whereas the angle of the AMIC was 163.6 degrees, the length of the DMS was $28.1 \mathrm{~mm}$ and CTL was $158 \mathrm{HU}$ in the non-haematoma side. It is obvious that the angle of the AMIC on the haematoma side was smaller than the angle of the AMIC on the non-haematoma side, the length of DMS on the haematoma side was much shorter than the length of DMS on the non-haematoma side, and the CTL on the haematoma side was greater than the CTL on the non-haematoma side. 


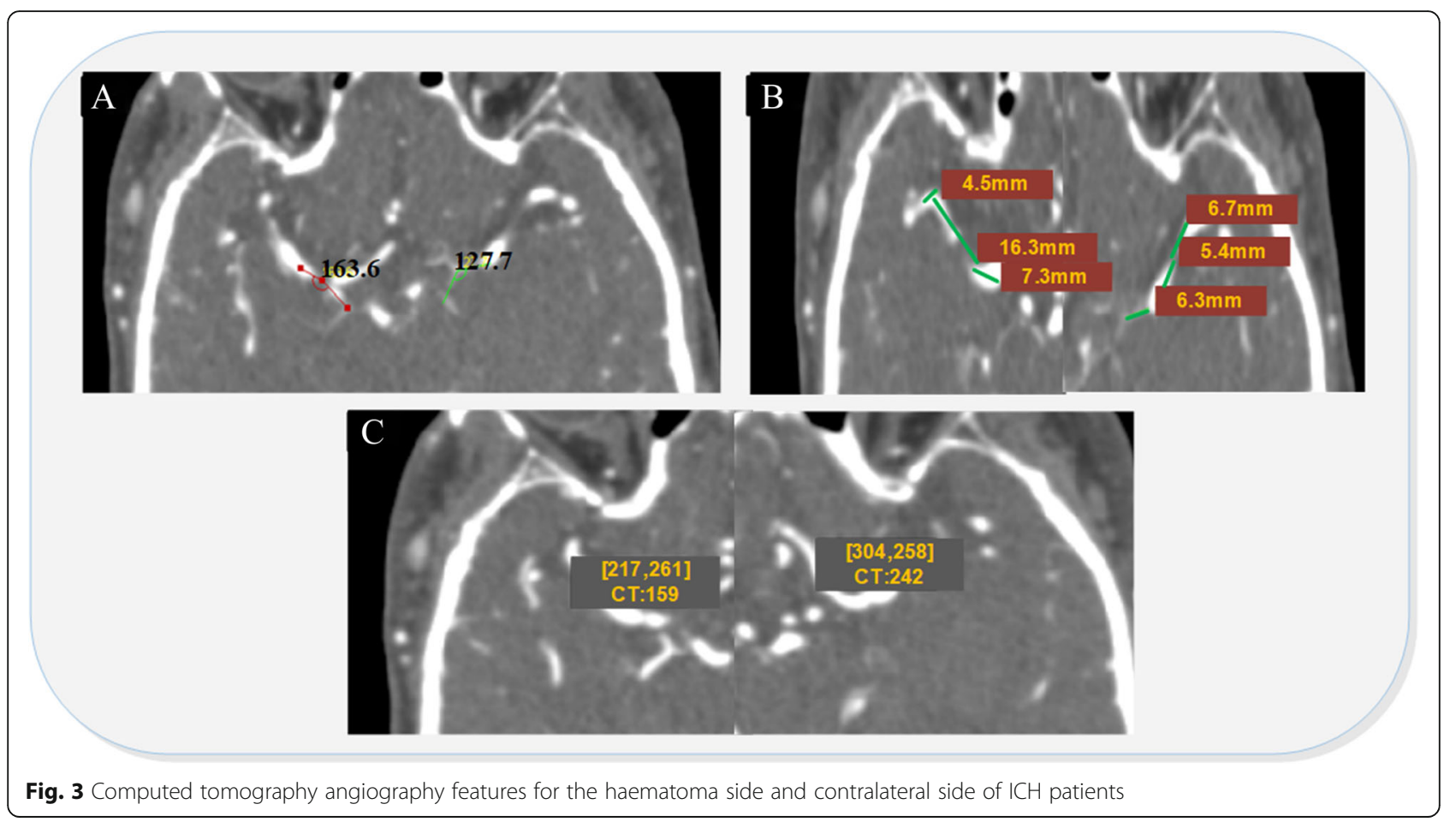

\section{Predictive performance for each model}

We employed SVM, decision tree, KNN and ensemble learning methods to develop the predictive model by using AMIC, DMS and CTL as the features.

Here, we employed cross validation [34] to train and test each model. We divided the data set into eight groups, 6 training sets and 2 test sets, to train and test the data. Figure 4a and Additional file 1: Table S7 show the sensitivity, specificity, precision and accuracy for $\mathrm{KNN}$, SVM, decision tree and ensemble learning, in which ensemble learning outperforms the other three. Additional file 1: Table S6 details four classic classification measurement standards (sensitivity, specificity, precision and accuracy).

Moreover, since the ensemble-learning algorithm employs the accuracy measurement as the objective function to optimize the key weight of the weak classifiers, here, we compare the predictive capacity among ensemble learning, SVM, KNN and decision tree in Fig. $4 \mathrm{~b}$ and compare the predictive capacity between ensemble-learning and previous research [20-22] in Fig. 4c. Figure $4 \mathrm{~b}$ and c demonstrate that ensemble learning performs best in the prediction of intracerebral haemorrhage based on the accuracy measurements with statistically significant results.

\section{Discussion}

Because of the high morbidity and mortality of intracerebral haemorrhage, the prevention and treatment of intracerebral haemorrhage is currently an issue of great concern. Research on the prediction of intracerebral haemorrhage is becoming increasingly important. Currently, the relative risk between intracerebral haemorrhage and hypertension, diabetes, hyperlipemia, or other systemic diseases is widely accepted, but these diseases correlate to other cardio-cerebral vascular incidents, such as ischaemic stroke and coronary heart disease, with poor specificity $[15,35]$. In clinical practice, it is still difficult to predict the direct haemorrhagic risk for these high-risk populations. There are many studies [15, 35 ] on the prediction of haematoma expansion after intracerebral haemorrhage by using CT images of the brain, such as spot signs. However, no other study has identified the haemorrhagic risk for the non-intracerebral haemorrhage potential patients, which may be the reason for the negative results of many intracerebral haemorrhage clinical trials by mixing these subpopulations with others. If so, risk identification might greatly facilitate precise control and prevention of the increasing occurrences of intracerebral haemorrhage [3].

$\mathrm{ICH}$ in the basal ganglion, which constitutes the majority of $\mathrm{ICH}$ subtypes that are frequently related to hypertensive vasculopathy, often occurs due to the rupture of small vessels, especially the branches of lenticulostriate artery, releasing the blood into the brain parenchyma [2]. The lenticulostriate artery usually arises from the trunk of the middle cerebral artery before the bifurcation. The intracerebral segments of the lenticulostriate artery with its branches are shaped as a curve or a loop, which results in much more pressure on the vessel wall when it flows through the bending portion. 


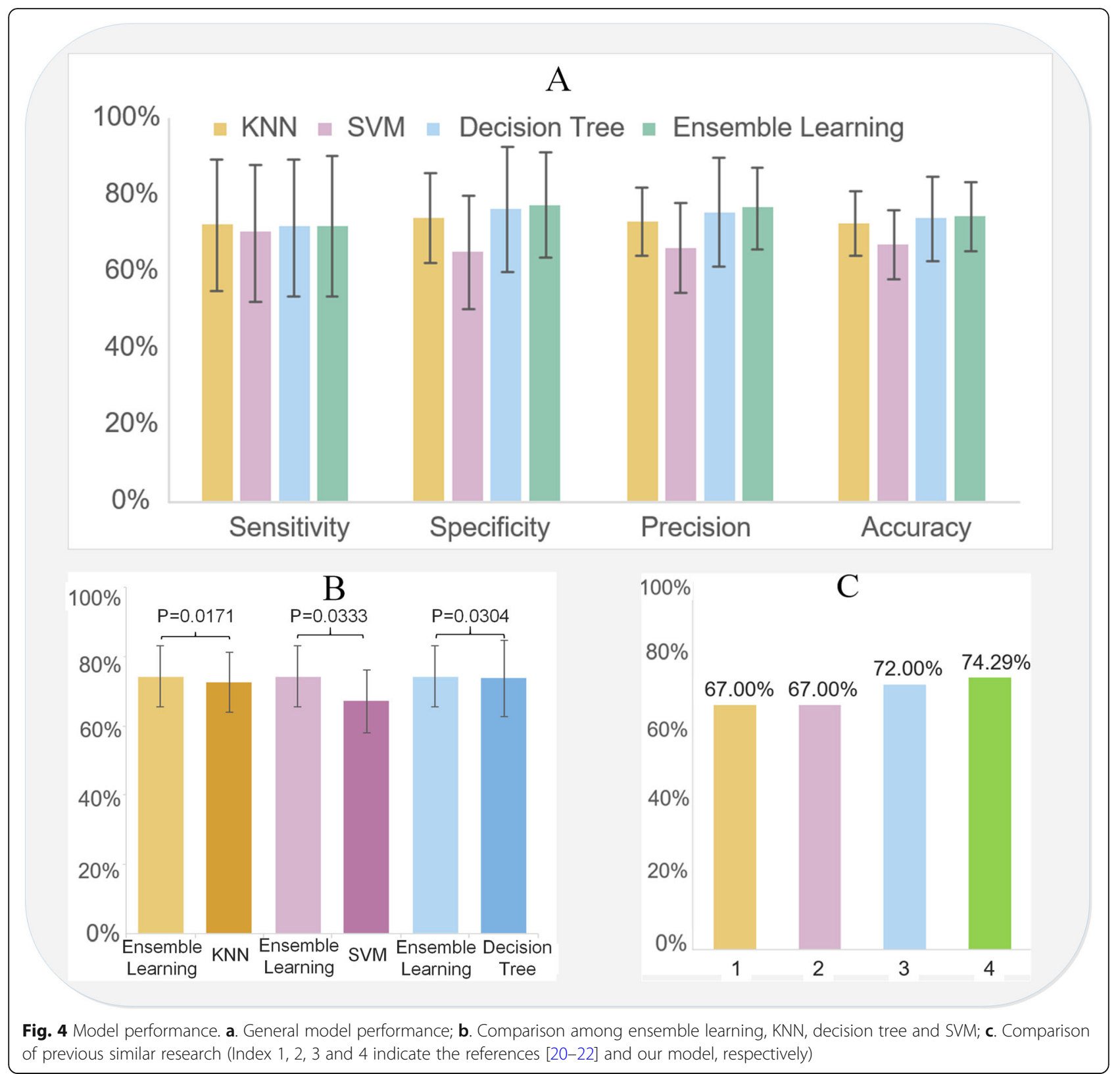

Therefore, the branches of the lenticulostriate artery bear much more shear stress or pressure due to the decreasing diameter and the unique morphology of the lenticulostriate artery and its branches, which becomes a high-risk factor for intracerebral haemorrhage [36, 37]. For this reason, the present study chose ten potential characteristics (Additional file 1: Table S3) associated with the shear stress of the lenticulostriate artery on computed tomography angiography imaging.

The present study found that the angle between the middle cerebral artery and the internal carotid artery (AMIC), the distance between the beginning of the median artery and superior trunk (DMS), and the CT of the lenticulostriate artery (CTL) are statistically significant enough to be causes of intracerebral haemorrhage (Additional file 1: Table S5). In addition, we employed these causes as the features of the classification model to predict the occurrence of intracerebral haemorrhage (Fig. 2). As we know, shear stress arises from the friction between blood flow and the vascular endothelium, paralleling the vessel wall. The magnitude of shear stress in straight vessels is directly proportional to the viscosity of blood and blood flow and inversely proportional to the third power of the inner radius of the vessel. The three characteristics we chose as features in the present study may be closely associated with the local shear stress around the basal ganglion, especially the density (CT value) of the lenticulostriate artery, as 
the greatest change in blood pressure and velocity of blood flow occurs at the transition from arterioles to capillaries. Briefly, the narrow AMIC (Fig. 3a) and short DMS (Fig. 3b) may lead to high impact forces towards the branches of the middle cerebral artery, while the high CT value in lenticulostriate arterial areas (CTL) illustrates tighter arterioles with possible weak vessel walls that receive the shear stress of the blood stream (Fig. 3c).

Moreover, since accuracy is an important measurement for clinical personnel [21] and the function of our ensemble-learning model, we compared the performance of the ensemble-learning method with previous similar studies and our three methods (KNN, decision tree and SVM), as shown in Fig. $4 \mathrm{~b}$ and c, respectively. Figure $4 \mathrm{~b}$ and $c$ demonstrate that these features are so efficient that our developed ensemble-learning method outperforms not only previous work [20-22] but also the other three methods based on accuracy measurements with statistically significant results. However, Fig. 4a shows that the sensitivity of ensemble learning was not better than that of the KNN method. Since the ensemble learning method employs accuracy as the objective function to optimize the key weights (Eq. 3 and Eq. 4) for each weak classifier, it cannot guarantee the best performance for the other three measurements, including sensitivity.

While this study demonstrated better performance in stroke prediction, it still has several drawbacks. For example, our sample size was too small for us to consider more classification measurements for the predictive model. Therefore, we are going to integrate more recent bioinformatics research algorithms [38-43] into this system and employ a large CTA imaging data set to overcome the current shortcomings.

In conclusion, AMIC, DMS and CTL can predict intracerebral haemorrhage. Our developed ensemble-learning method efficiently employs these selected features to efficiently predict the probability of intracerebral haemorrhage.

\section{Conclusions}

In this article, we propose a mathematical framework for the prediction of intracerebral haemorrhage. Statistical methods are employed to compute the size of the samples and demonstrate which candidate biomarkers are statistically significant enough to be the features of the model. A novel ensemble-learning method is developed by integrating SVM, decision tree and KNN algorithms into the model to improve the predictive accuracy. Our research results reveal that AMIC, DMS and CTL can predict intracerebral haemorrhage, and the developed mathematical model in the present study not only outperforms the classic KNN, decision tree and SVM models but also performs better than previous similar research.

\section{Additional file}

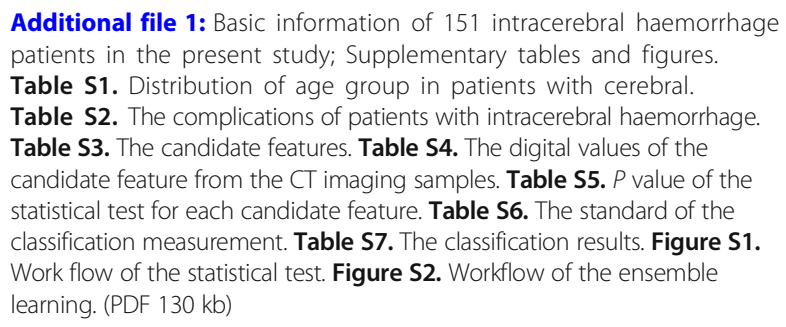

Abbreviations

KNN: K-Nearest Neighbour; SVM: Support Vector Machine

\section{Acknowledgements}

Not applicable.

\section{Funding}

Publication costs are funded by the Major Innovation Project of Southwest Hospital (No. SWH2016ZDCX1011), the National Basic Research Program of China (No. 2014CB541600), National Science and Technology Major Project of China (NO. 2018ZX10201002) and the National Natural Science Foundation of China (NO. 81501002 and NO.61372138).

\section{Availability of data and materials}

All data generated or analysed during this study are included in this published article and its supplementary information files.

\section{About this supplement}

This article has been published as part of BMC Bioinformatics Volume 20 Supplement 7, 2019: Selected papers from the 12th International Conference on Computational Systems Biology (ISB 2018). The full contents of the supplement are available online at https://bmcbioinformatics.biomedcentral.com/articles/ supplements/volume-20-supplement-7.

\section{Authors' contributions}

$\mathrm{LZ}$ and $Y J C$ conceived the study and developed the model. $J \mathrm{~L}$ and $K K Y$ performed the simulations for the model. ZYJ and TTL wrote the manuscript. $\mathrm{RH}$ and $\mathrm{ZY}$ performed the analysis for the model. HF contributed to acquisition of data. All authors read and approved the final manuscript.

\section{Ethics approval and consent to participate}

A total of 151 patients with unilateral hypertensive $\mathrm{ICH}$ admitted to the Department of Neurosurgery, Southwest Hospital, Chongqing, China and confirmed with computed tomography angiography between January 2012 and December 2016 were analysed retrospectively. The protocol was approved by the Ethics Committee of Southwest Hospital, and the committee waived the need for patient consent due to the retrospective nature of the study.

\section{Consent for publication \\ Not applicable.}

\section{Competing interests}

The authors declare that they have no competing interests.

\section{Publisher's Note}

Springer Nature remains neutral with regard to jurisdictional claims in published maps and institutional affiliations.

\section{Author details}

${ }^{1}$ College of Computer and Information Science, Southwest University, Chongqing 400715, People's Republic of China. ${ }^{2}$ College of Computer Science, Sichuan University, Chengdu 610065, People's Republic of China. ${ }^{3}$ Medical Big Data Center, Sichuan University, Chengdu 610065, People's Republic of China. ${ }^{4}$ School of Medical Information and Engineering, Southwest Medical University, Luzhou 646000, People's Republic of China. 
${ }^{5}$ Department of Neurosurgery, Southwest Hospital, Third Military Medical University, Chongqing 400038, People's Republic of China. ${ }^{6}$ School of Mathematics and Statistics, Southwest University, Chongqing 400715, People's Republic of China. ${ }^{7}$ Department of Neurosurgery, Fuling Central Hospital, Chongqing 400715, People's Republic of China.

\section{Published: 1 May 2019}

\section{References}

1. Feigin VL, Forouzanfar MH, Krishnamurthi R, Mensah GA, Connor M, Bennett DA, Moran AE, Sacco RL, Anderson L, Truelsen T, et al. Global and regional burden of stroke during 1990-2010: findings from the global burden of disease study 2010. Lancet. 2014;383(9913):245-54.

2. Qureshi Al, Tuhrim S, Broderick JP, Batjer HH, Hondo H, Hanley DF. Spontaneous intracerebral hemorrhage. N Engl J Med. 2001;344(19): 1450-60.

3. Wang W, Jiang B, Sun $H$, Ru X, Sun D, Wang L, Wang L, Jiang Y, Li Y, Wang $Y$, et al. Prevalence, incidence, and mortality of stroke in China: results from a Nationwide population-based survey of 480687 adults. Circulation. 2017; 135(8):759-71.

4. $\mathrm{Xi} \mathrm{G}$, Strahle J, Hua Y, Keep RF. Progress in translational research on intracerebral hemorrhage: is there an end in sight? Prog Neurobiol. 2014; 115:45-63.

5. de Rooij NK, Greving JP, Rinkel GJ, Frijns CJ. Early prediction of delayed cerebral ischemia after subarachnoid hemorrhage: development and validation of a practical risk chart. Stroke. 2013:44(5):1288-94.

6. Harrod CG, Bendok BR, Batjer HH. Prediction of cerebral vasospasm in patients presenting with aneurysmal subarachnoid hemorrhage: a review. Neurosurgery. 2005;56(4):633.

7. Hijdra A, Gijn JV, Nagelkerke NJ, Vermeulen M, Crevel HV. Prediction of delayed cerebral ischemia, rebleeding, and outcome after aneurysmal subarachnoid hemorrhage. Stroke. 1988;19(10):1250.

8. Kusano Y, Seguchi T, Horiuchi T, Kakizawa Y, Kobayashi T, Tanaka Y, Seguchi $\mathrm{K}$, Hongo K. Prediction of functional outcome in acute cerebral hemorrhage using diffusion tensor imaging at $3 \mathrm{~T}$ : a prospective study. AJNR Am J Neuroradiol. 2009;30(8):1561-5.

9. Sridharan SE, Unnikrishnan JP, Sukumaran S, Sylaja PN, Nayak SD, Sarma PS, Radhakrishnan K. Incidence, types, risk factors, and outcome of stroke in a developing country: the Trivandrum stroke registry. Stroke. 2009;40(4):1212-8.

10. Xin WANG, XW-Y XIAO H-z. The correlation analysis of cerebral hemorrhage and meteorological factors and its prediction study in Jingmen City. Chin J Prev Contr Chron Dis. 2012;20(5):557-9.

11. Aimei ea L. Relationship between meteorological factors and cardiocerebrolvasular diseases incidence. Chinese Journal of Prevention and Control of Chronic Non-Communicable Diseases. 1997:(2):61-3.

12. Lukic S, Cojbasic Z, Peric Z, Milosevic Z, Spasic M, Pavlovic V, Milojevic A. Artificial neural networks based early clinical prediction of mortality after spontaneous intracerebral hemorrhage. Acta Neurol Belg. 2012; 112(4):375-82.

13. Li YF, Luo J, Li Q, Jing YJ, Wang RY, Li RS. A new simple model for prediction of hospital mortality in patients with intracerebral hemorrhage. CNS Neurosci Ther. 2012;18(6):482-6.

14. Cao D, Li Q, Fu P, Zhang J, Yang J. Early hematoma enlargement in primary intracerebral hemorrhage. Curr Drug Targets. 2017;18(12):1345.

15. Li Q, Huang YJ, Zhang G, Lv FJ, Wei X, Dong MX, Chen JJ, Zhang L, Qin XY, Xie P. Intraventricular hemorrhage and early hematoma expansion in patients with intracerebral hemorrhage. Sci Rep. 2015;5:11357.

16. Dietterich TG. Ensemble methods in machine learning, vol. 1857; 2000. p. 1): $1-15$.

17. Burges CJC: Simplified support vector decision rules. In Proceedings of the thirteenth international conference on international conference on machine learning. Morgan Kaufmann Publishers Inc. 1996. 71-77.

18. Quinlan JR. Induction on decision tree. Mach Learn. 1986;1(1):81-106.

19. Cover T, Hart P. Nearest neighbor pattern classification. IEEE Trans Inf Theory. 1967;13(1):21-7.

20. SHEN Mingyang SX. The progess of clinical applications of the computed tomography angiography spot sign in hypertensive intracerebral hemorrhage. Anhui Medical and Pharmaceutical Journal. 2017;21(6):1127-9.

21. Almandoz JED, Kelly HR, Schaefer PW, Brouwers HB, Yoo AJ, Stone MJ, Goldstein JN, Rosand J, Lev MH, Gonzalez RG. CT angiography spot sign predicts in-hospital mortality in patients with secondary intracerebral hemorrhage. Journal of Neurointerventional Surgery. 2012;4(6):442-7.

22. Brouwers HB, Falcone GJ, Mcnamara KA, Ayres AM, Oleinik A, Schwab K, Romero JM, Viswanathan A, Greenberg SM, Rosand J. CTA spot sign predicts hematoma expansion in patients with delayed presentation after intracerebral hemorrhage. Neurocrit Care. 2012;17(3):421-8.

23. Hu R, Feng $\mathrm{H}$. Lenticulostriate artery and Lenticulostriate-artery neural complex: new concept for intracerebral hemorrhage. Curr Pharm Des. 2017; 23(15):2206-11.

24. Qureshi Al. The importance of acute hypertensive response in $\mathrm{ICH}$. Stroke. 2013:44(6 Suppl 1):S67-9.

25. Oeinck M, Neunhoeffer F, Buttler K, Meckel S, Schmidt B, Czosnyka M, Weiller $C$, Reinhard M. Dynamic cerebral autoregulation in acute intracerebral hemorrhage. Stroke. 2013;44(10):2722-8.

26. Kabacoff R: R in action. Manning Publications Co.; 2011.

27. Wang R. Medical experiment design and statistical analysis. Beijing: Beijing Medical University Press; 2000

28. Lau B, Marionalbares: Process: Pre Matlab release 2014. 2015.

29. Dietterich TG. Machine learning research: four current directions. Al Mag. 2000;18(4):97-136.

30. Wu X, Kumar V, Quinlan JR, Ghosh J, Yang Q, Motoda H, Mclachlan GJ, Ng A, Liu B, Yu PS. Top 10 algorithms in data mining. Knowl Inf Syst. 2008;14(1):1-37.

31. Zahuranec DB, Sanchez BN, Brown DL, Wing JJ, Smith MA, Garcia NM, Meurer WJ, Morgenstern LB, Lisabeth LD. Computed tomography findings for intracerebral hemorrhage have little incremental impact on post-stroke mortality prediction model performance. Cerebrovasc Dis. 2012;34(1):86-92.

32. Adeoye O, Broderick JP. Advances in the management of intracerebral hemorrhage. Nat Rev Neurol. 2010;6(11):593-601.

33. Zhang L, Zheng C, Li T, Xing L, Zeng H, Li T, Yang H, Cao J, Chen B, Zhou Z. Building up a robust risk mathematical platform to predict colorectal cancer. Complexity. 2017;2017(8):1-14.

34. Kohavi R. A study of cross-validation and bootstrap for accuracy estimation and model selection. In: Proceedings of international joint conference on artificial intelligence. San Fransisco: Morgan Kaufmann Publishers Inc; 1995. p. $1137-43$.

35. Sorimachi T, Osada T, Baba T, Inoue G, Atsumi H, Ishizaka H, Hotta K, Hayashi N, Matsumae M. The striate artery, hematoma, and spot sign on coronal images of computed tomography angiography in putaminal intracerebral hemorrhage. Stroke. 2013:44(7):1830-2.

36. Chien S, Li S, Shyy YJ: Effects of mechanical forces on signal transduction and gene expression in endothelial cells. Hypertension 1998, 31 (1 Pt 2):162-169.

37. Chiu JJ, Chien S. Effects of disturbed flow on vascular endothelium: pathophysiological basis and clinical perspectives. Physiol Rev. 2011;91(1): 327-87.

38. Zhang L, Zhang S. Using game theory to investigate the epigenetic control mechanisms of embryo development: comment on: "epigenetic game theory: how to compute the epigenetic control of maternal-to-zygotic transition" by Qian Wang et al. Phys Life Rev. 2017;20:140-2.

39. Zhang L, Liu Y, Wang M, Wu Z, Li N, Zhang J, Yang C. EZH2-, CHD4- and IDH-linked epigenetic perturbation and its association with survival in glioma patients. J Mol Cell Biol. 2017;9(6).

40. Xia Y, Yang C, Hu N, Yang Z, He X, Li T, Zhang L. Exploring the key genes and signaling transduction pathways related to the survival time of glioblastoma multiforme patients by a novel survival analysis model. BMC Genomics. 2017;18(Suppl 1):950.

41. Li T, Cheng Z, Zhang L. Developing a novel parameter estimation method for agent-based model in immune system simulation under the framework of history matching: a case study on influenza a virus infection. Int J Mol Sci. 2017;18(12)

42. Gao H, Yin Z, Cao Z, Zhang L. Developing an agent-based drug model to investigate the synergistic effects of drug combinations. Molecules. 2017; 22(12):2209

43. Zhang L, Qiao M, Gao H, Hu B, Tan H, Zhou X, Li CM. Investigation of mechanism of bone regeneration in a porous biodegradable calcium phosphate (CaP) scaffold by a combination of a multi-scale agent-based model and experimental optimization/validation. Nanoscale. 2016;8(31): 14877. 